\title{
Comparison between Medical Student Educators and Program Directors in 2010 and 2019
}

\author{
Prithvi S. Sankar, MD ${ }^{1}$ JoAnn A. Giaconi, MD ${ }^{2}$ Anju Goyal, MD ${ }^{3}$ Emily G. Graubart, MD \\ Pavlina S. Kemp, MD ${ }^{5}$ Daniel W. Knoch, MD Jamie B. Rosenberg, MD ${ }^{7}$ \\ ${ }^{1}$ Scheie Eye Institute, Department of Ophthalmology, University of \\ Address for correspondence Prithvi S. Sankar, MD, Scheie Eye \\ Pennsylvania, Philadelphia, Pennsylvania \\ 2 Stein Eye Institute, David Geffen School of Medicine, University of \\ Institute, 51 North 39th Street, Philadelphia, PA 19104 \\ California at Los Angeles, Los Angeles, California \\ 3 Kresge Eye Institute, Department of Ophthalmology, Visual and \\ Anatomical Sciences, Wayne State University, Detroit, Michigan \\ ${ }^{4}$ Department of Ophthalmology, Emory University School of \\ Medicine, Atlanta, Georgia \\ ${ }^{5}$ Department of Ophthalmology and Visual Sciences, University of \\ lowa Hospitals and Clinics, lowa City, lowa \\ ${ }^{6}$ Department of Ophthalmology and Visual Sciences, University of \\ Wisconsin School of Medicine and Public Health, Madison, Wisconsin \\ ${ }^{7}$ Department of Ophthalmology and Visual Sciences, Montefiore \\ Medical Center / Albert Einstein College of Medicine, Bronx, \\ New York \\ (e-mail: prithvi.sankar@pennmedicine.upenn.edu). \\ J Acad Ophthalmol 2021;13:e264-e269.
}

\begin{abstract}
Keywords

- director medical student education

- program director

- academic promotion

- women in medicine
\end{abstract}

Objective Over the last decade, prominence of the Directors of Medical Student Education in Ophthalmology (DMSEs) within the Association of University Professors of Ophthalmology (AUPO) has increased. With increased recognition of this important leadership position, an examination of the trending demographic differences between DMSEs and the Ophthalmology Program Directors (PDs) may be helpful, especially in regard to the potential for academic promotion from each track.

Design Present study is a database study.

Methods The AUPO directory was used to ascertain the DMSE and the PD members between 2010 and 2019. The demographic profile for each member was determined using publicly available resources. Chi-square analysis of the data was performed.

Main Outcome Measures Number of DMSE and PD AUPO from 2010 to 2019 in AUPO Directory, sex, geographic location, and graduation year were measured through this study. Results There has been a steady increase in the number of DMSEs from 2010 to 2019, whereas the number of PDs have remained stable. The number of DMSEs have increased in all regions of the United States. The year of ophthalmology residency graduation was similar in the DMSE and PD cohorts in 2010 and 2019. The number of women have increased in the DMSEs in 2019 compared with 2010; the number of women who were PDs in 2019 were similar to 2010. Finally, more PDs in 2010 became Chairs in 2019, whereas no DMSEs in 2010 became Chair in 2019. received

February 13, 2021 accepted after revision August 19, 2021
DOI https://doi.org/ 10.1055/s-0041-1740064. ISSN 2475-4757.

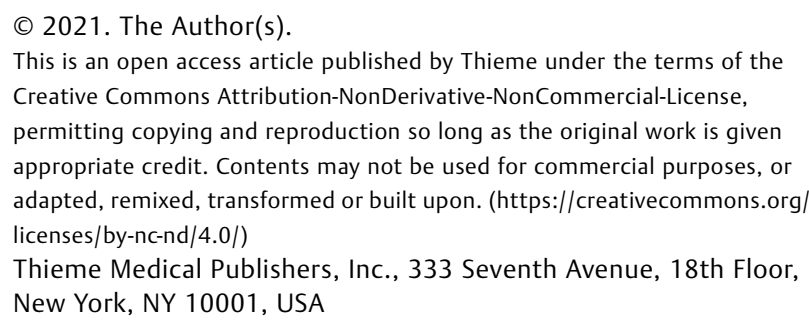
appropriate credit. Contents may not be used for commercial purposes, or adapted, remixed, transformed or built upon. (https://creativecommons.org/ licenses/by-nc-nd/4.0/) Thieme Medical Publishers, Inc., 333 Seventh Avenue, 18th Floor, New York, NY 10001, USA 
Conclusion There are a growing number of DMSEs, overall, with an increasing proportion of women in the 2019 DMSE group compared with 2010. In contrast, the overall number of PDs have remained stable, as has the proportion of female PDs in 2019 compared with 2010. DMSEs, compared with PDs, are less likely to become Chairs. The increased opportunity for academic leadership with the growing DMSE group may help change leadership patterns overall in academic ophthalmology.

Within ophthalmology, Directors of Medical Student Education (DMSEs) and Program Directors (PDs) are dedicated to teaching, implementing, and administering educational programs that train future physicians and ophthalmologists in particular. The Association of University Professors of Ophthalmology (AUPO) is an organization that serves, strengthens, and represents academic departments of ophthalmology. ${ }^{1}$ Until recently, the members of the AUPO were the Chairs of the Departments of Ophthalmology in the United States and Canada. Currently, many departmental leaders, including DMSEs and PDs are also members. The DMSEs were added in $2010^{1}$ in an effort to reverse the decline in ophthalmology education for medical students that has left the majority of medical schools without dedicated time for ophthalmology in their curriculum. ${ }^{2-5}$

Since 2010, the demographics of ophthalmology educational leadership has changed and continues to evolve. A 2018 study revealed that $90 \%$ of the Chairs of ophthalmology are men but with a slight trend of recent female Chair appointments. ${ }^{6}$ Historically, being a PD has been seen as a pathway to leadership within a medical department, whereas being a DMSE has not been seen as a steppingstone to further academic leadership within a department. ${ }^{7}$ The purpose of this study is to analyze the data from the AUPO registry to specifically determine the similarities and differences in demographic trends for DMSEs and PDs from 2010 to 2019.

\section{Methods}

Institutional Review Board approval was requested and exemption was granted by the University of Pennsylvania.
The AUPO directory was used to identify the Chairs, DMSEs, and PDs inclusive of the years 2010 to 2019. Using publicly available resources, including the AUPO member directory, program web sites, and newsletters, the American Academy of Ophthalmology members' directory, online curriculum vitae, and state and national physician registries, data were collected on each member. Data included the year of residency graduation and gender. The United States Census Bureau definition of geographic distribution was used to assign programs to a particular region. ${ }^{8}$

Data were collected and analysis was done using Microsoft Excel 2016 (Microsoft Inc., Redmond, WA). Statistical analysis was used using excel, as well as an online statistical calculator. ${ }^{9}$ Statistics using $\chi^{2}$ were done with a significance level of 0.05 .

\section{Results}

The number of DMSEs have increased year to year from 39 in 2010 to 66 in 2019. The number of PDs have essentially remained the same year to year in the same time period, 101 compared with 102, respectively (-Fig. 1). The number of DMSEs have grown in all U.S. regions, while the overall PD numbers in geographic areas have been stable, except for a small increase in the number of PDs in the Northeast with a compensatory decrease in the South (-Fig. 2). Canadian programs only demonstrated an increase in the PD group (from 1 in 2010-3 in 2019) without an increase in DMSE (with 1 in 2010 and 2019). These changes were not statistically significant.

The years of graduation from ophthalmology residency for the DMSE and PD groups from 2010 and 2019 were

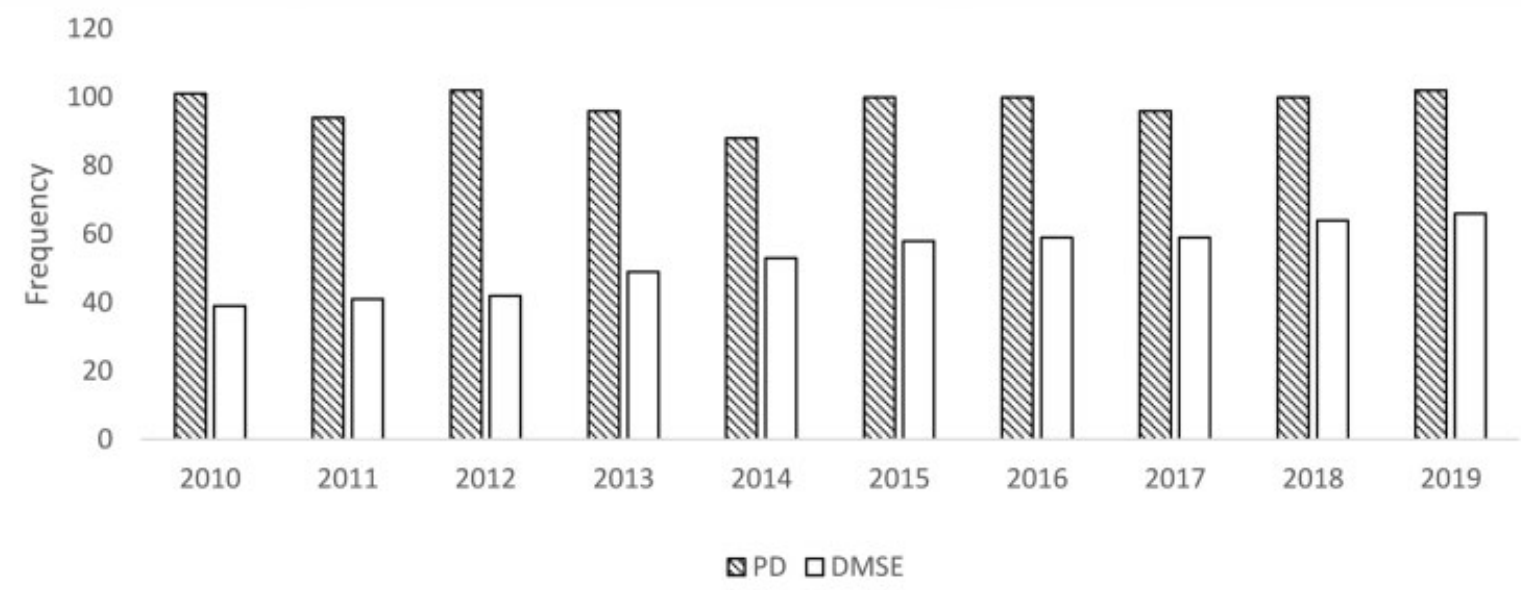

Fig. 1 The number of DMSE and PD from 2010 to 2019. The number of DMSEs have increased from 2010-2019. The number of PDs have remained essentially the same. DMSE, Director of Medical Student Education in Ophthalmology; PD, Program Director. 


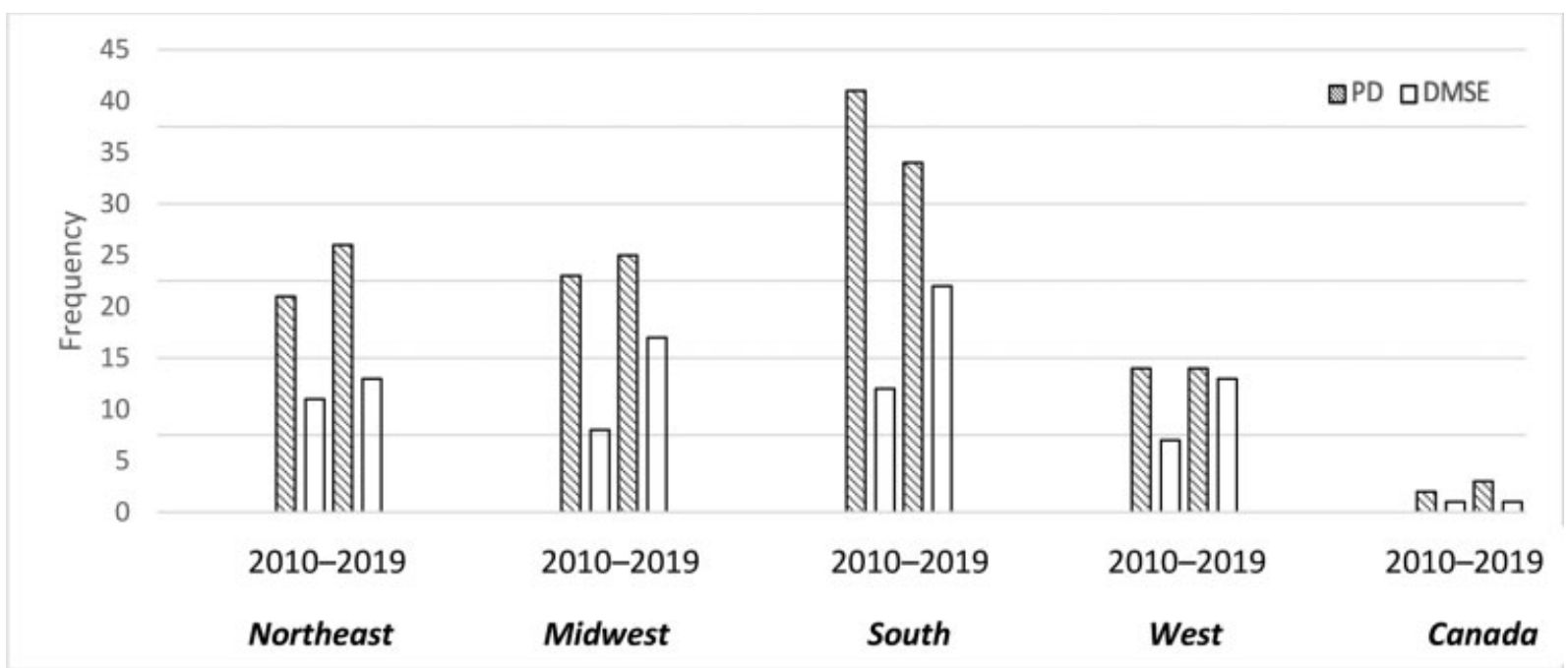

Fig. 2 The geographic distribution of DMSE and PD in 2010 and 2019. The number of DMSEs have increased in all geographic regions from 2010-2019, while the number of PDs have remained essentially the same in all regions. DMSE, Director of Medical Student Education in Ophthalmology; PD, Program Director.

analyzed. Only the graduation years of ophthalmology residencies were obtained. If an individual graduated from multiple medical specialties (e.g., internal medicine and ophthalmology) only the year of graduation from ophthalmology residency was counted.

There was a broad distribution in the graduation year for the DMSEs in 2010 (-Fig. 3A). The span ranged from 1960 $(n=1)$ to $2008(n=1)$. The greatest number of DMSEs gradu- ated in $1981,1998,1999$, and 2000 ( $n=5$ in each graduation year). - Fig. 3B demonstrates the graduation years of the DMSEs in 2019, suggesting a trend toward more recent graduates. For the PDs in 2010, the position was most frequently held by individuals who had graduated 16 years earlier in 1994, with a bell curve of graduation years on either side. Similar to the DMSEs in 2019, the PDs in 2019 had a trend toward more recent graduates occupying the position.

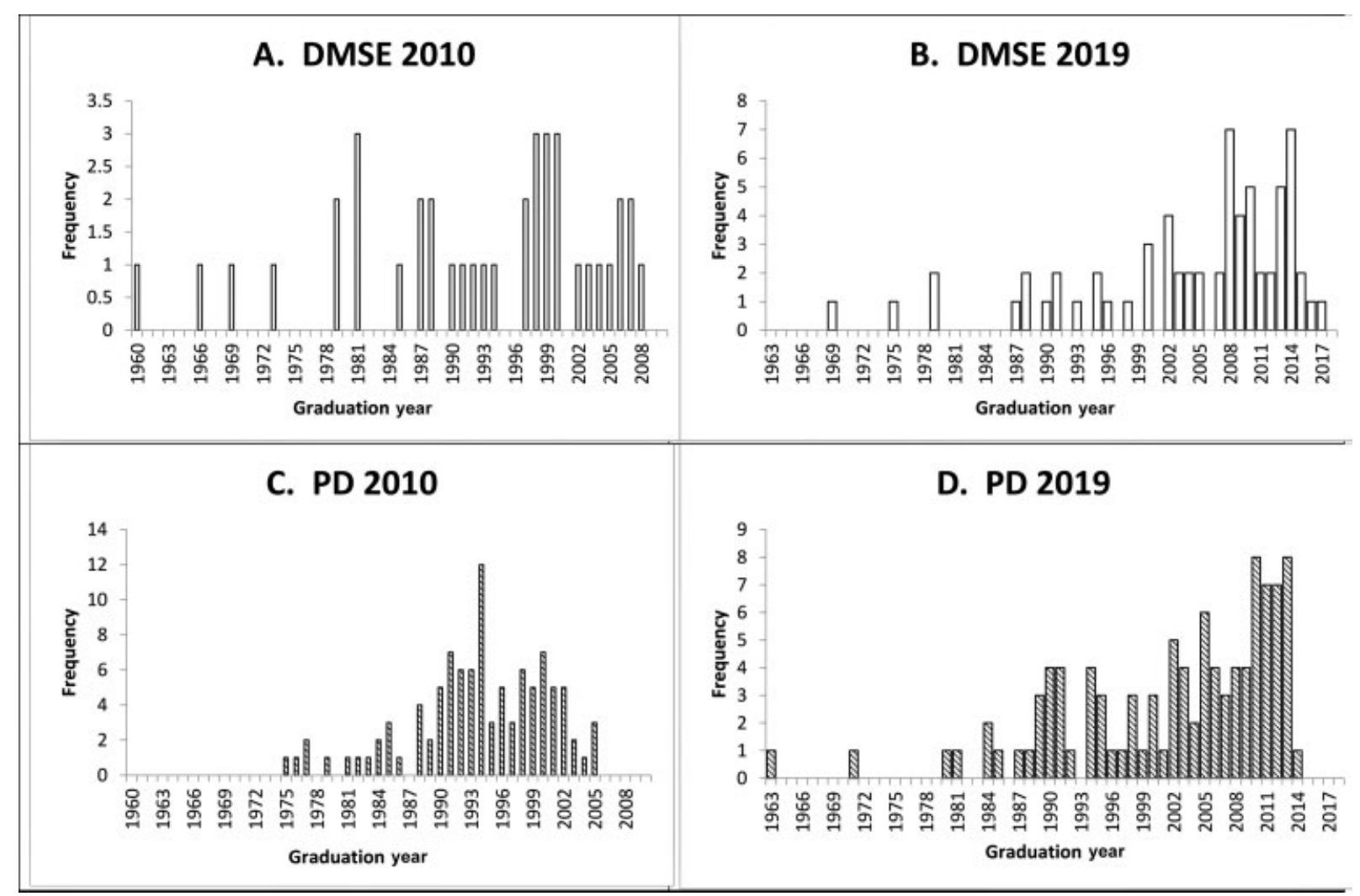

Fig. 3 Residency graduation years for DMSE and PD in 2010 and 2019. The graduation year for (A) DMSE in 2010; (B) DMSE in 2019; (C) PD in 2010; and (D) PD in 2019. DMSE, Director of Medical Student Education in Ophthalmology; PD, Program Director. 


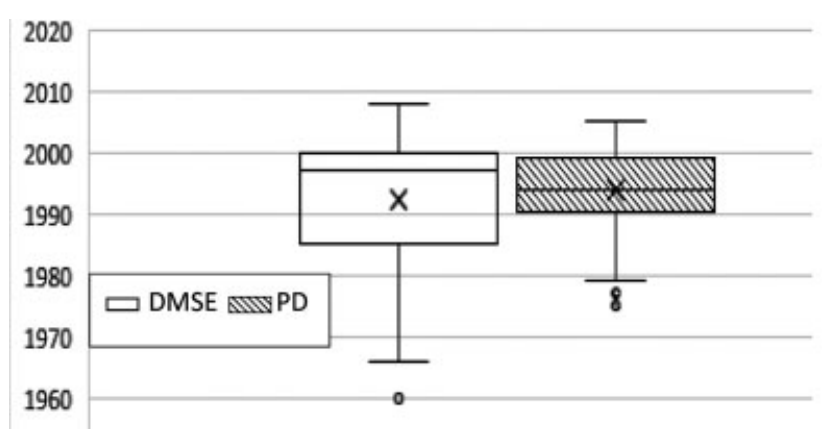

A

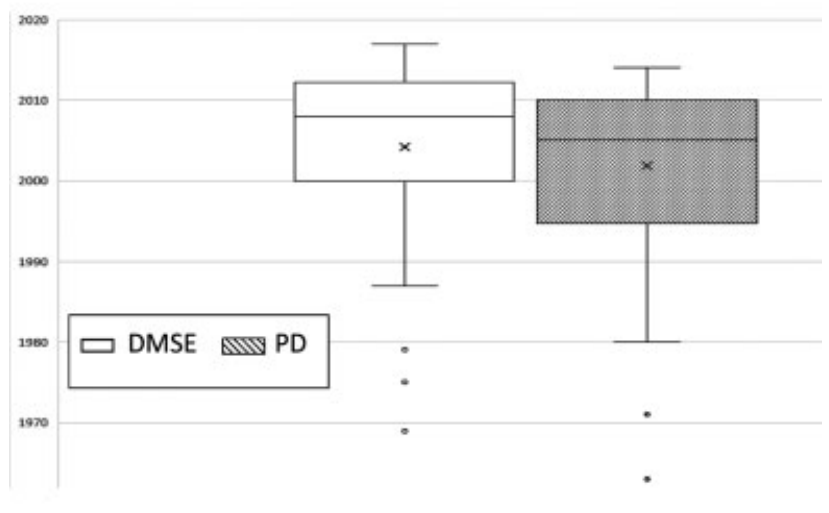

B

Fig. 4 The median graduation year for the DMSE and PD groups in (A) 2010 and (B) 2019. (A) In 2010, the median for the DMSE was 1997, the mean $(X)$ was 1992 and the IQR was 1986-2000. The median for the PD was 1994 with a mean (X) of 1993 and an IQR of 1991-1999. (B) In 2019, the median for the DMSE was 2008, the mean (X) was 2004 and the IQR was 2000-2012. The median for the PD was 2005 with a mean (X) of 2002 and an IQR of 1995-2010. DMSE, Director of Medical Student Education in Ophthalmology; IQR, interquartile range; PD, Program Director.

The data between the two groups in 2010 are more directly compared in - Fig. 4A. The median residency graduation year for the DMSEs was 1997 versus 1994 for the PDs. The median was used in this analysis since there were a few individuals in both groups who graduated well outside the confidence intervals. Similarly, the comparisons between the DMSEs and PDs in 2019 in - Fig. 4B demonstrate a difference in the medians between the two groups (2008 vs. 2005, respectively). Although in general, the DMSE graduated more recently than the PD in both the 2010 and 2019 cohorts, there was no statistically significant difference.

Gender was ascertained for each individual in the 2010 cohort and the 2019 cohort using the same data sources (-Table 1) and differences across groups were compared. - Table 1A demonstrates the DMSEs and PDs in 2010. In 2010, there were more men in both groups, $(p=0.0006)$, but there was no statistically significant difference in the proportion of women who were DMSEs and the proportion of women who were PDs $(p=0.17)$.

As shown in - Table 1B, in 2019, there was a statistically significant difference between the number of women in each group, with more women in the DMSE group and more men
Table 1 The number of women and men in the DMSE and PD groups

\begin{tabular}{|c|c|c|}
\hline \multicolumn{3}{|c|}{$\begin{array}{l}\text { A. Distribution of gender in the DMSE and PD group in } \\
2010\end{array}$} \\
\hline & DMSE & PD \\
\hline Women & 15 & 27 \\
\hline Men & 24 & 74 \\
\hline
\end{tabular}

Percentage of DMSE women compared with percentage of PD women, $X^{2}(1, n=140)=1.8, p=0.17$

Total number of women compared with the number of males, $X^{2}(1, n=140)=11.67, p=0.0006$

\begin{tabular}{|l|l|l|}
\hline \multicolumn{3}{|l|}{ B. Distribution of gender in the DMSE and PD group in 2019} \\
\hline Women & DMSE & PD \\
\hline Men & 40 & 26 \\
\hline Pen & 26 & 76 \\
\hline
\end{tabular}

Percentage of DMSE women compared with percentage of PD women, $X^{2}(1, n=168)=20.7, p \leq 0.00001$

Total number of women compared with the number of men, $X^{2}(1, n=168)=3.0, p=0.048$

C. Distribution of gender in the DMSE groups in 2010 and 2019

\begin{tabular}{|l|l|l|}
\hline & 2010 & 2019 \\
\hline Women & 15 & 40 \\
\hline Men & 24 & 26 \\
\hline Percentage of women in 2010 compared with 2019, & (1, \\
\hline
\end{tabular}

Percentage of women in 2010 compared with $2019, X^{2}(1$, $n=105)=4.8, p=0.028$

D. Distribution of gender in the PD groups in 2010 and 2019

\begin{tabular}{|l|l|l|}
\hline & 2010 & 2019 \\
\hline Women & 27 & 26 \\
\hline Men & 74 & 76 \\
\hline Percentage
\end{tabular}

Percentage of women in 2010 compared with $2019, x^{2}(1$, $n=203)=0.04, p=0.84$

Abbreviations: DMSE, Director of Medical Student Education in Ophthalmology; PD, Program Director.

in the PD group, $(p \leq 0.00001)$. These numbers represent a statistical increase in the proportion of women in the DMSE group, $p=0.028$ ( - Table 1C), without a similar change in the PD group, $(p=0.84$; - Table 1D).

The total numbers of associate members (DMSEs and PDs) increased from 2010 to 2019, particularly in the number of DMSEs and women. There remained a statistically significant difference in the number of men compared with women in 2019 ( $p=0.048$ ), but it was less significant than in 2010 .

When looking at the movement between groups, two DMSEs in 2010 were PD in 2019 and one PD in 2010 was a DMSE in 2019. When we looked at the list of the Chairs, 14 PDs in 2010 were Chairs in 2019; however, no DMSE in 2010 was a Chair in 2019.

\section{Discussion}

The purpose of this study is to analyze the demographics, training, and characteristics of the ophthalmology DMSEs 
and PDs. We believe, this is the first study to look at the differences between DMSEs and PDs in any field.

Since 2010, there has been an increase in the number of DMSEs in the AUPO, while the number of PDs have remained the same, as seen in - Fig. 1. There are many explanations for this difference. The number of residency positions are governed by the 1997 Balance Budget Act by Congress. ${ }^{10}$ Having a program director is a required element for a residency program and the number of residency programs have essentially not changed, therefore the number of PDs has remained stable. Since ophthalmology is not a required clerkship in all medical schools, ${ }^{3-5}$ there is a wide variety of ophthalmology offerings to students and no regulation to have a DMSE. Overall, there has been an increase in DMSEs from 2010 to 2019, with an increase in most geographic areas. As noted, the DMSEs have been officially included in the AUPO as associate members since 2010 and now recently denoted as members since 2019 . This inclusion has raised more awareness of the importance of having a DMSE, contributing to the steady increase in the number of DMSE from 2010 to 2019.

In terms of the proximity of residency graduation year to holding the DMSE and PD position in 2010 or 2019, the data showed similar trends between cohorts. In the 2010 PD cohort, there was a peak in the number of 1994 residency graduates holding the position, with most program directors graduating within 6 to 8 years on either side of 1994. The DMSE 2010 cohort did not show any consistency in graduation year. However, there was no statistical difference between the two groups. In both the 2019 DMSE and PD cohorts, more recent graduates held these positions, as seen in - Fig. 3; and again there was no difference between the DMSE and PD groups in 2019. This change from 2010 to 2019 may be attributable to turnover in each group and the increase in new DMSEs. We decided to use the year of ophthalmology residency graduation as opposed to the year graduating medical school for a variety of reasons. Many individuals may not have entered ophthalmology residency immediately after graduating medical school, including those who may have graduated from foreign medical schools. Also, there were individuals who graduated from another residency prior to entering ophthalmology residency. Once ophthalmology training was completed, we felt that the career trajectory was more definitive. Fellowship training for the DMSE and PD was more challenging to ascertain from public records and therefore was not included. The Accreditation Council for Graduate Medical Education (ACGME) dictates that individuals cannot assume the position of PD any earlier than 3 years after completion of a residency, whereas there is no minimum timeline requirement to become a DMSE. ${ }^{11}$

Gender differences over time have shown the greatest change as seen in - Table 1. In 2010, the proportion of women to men in each group was not statistically significant between the DMSE and PD groups; however, there were statistically significant fewer women than men in both groups. This mirrors other specialties which have had low percentages of women in the PD role. ${ }^{12}$ In 2019 , there was a statistical increase in the proportion of women in the DMSE group but no change was seen in the PD cohort. The total number of women did increase from 2010 to 2019 in the aggregate of DMSE and PD, solely due to the increase in women DMSEs. In general, women are more underrepresented in the ophthalmology workforce and specifically in academic ophthalmology. Even so, there has been a gradual increase in the percentage of women in recent years. ${ }^{13,14}$ This observation is consistent with national trends in other medical specialties. Furthermore, there has been a recent trend for women to hold more leadership roles PD in a department. ${ }^{15,16}$

Finally, the data show that 14 PDs in 2010 were Chairs of ophthalmology in 2019, but no 2010 DMSEs were Chairs in 2019. This data are cross-sectional rather than longitudinal. We cannot state that there is no DMSE in 2010 who became Chair prior to 2019 but then was not a Chair in 2019. Nonetheless, the data suggest that being a PD was more favorable to becoming a chair of ophthalmology than being a DMSE, though this may not be typical for every specialty in medicine. $^{17}$

\section{Limitations}

This study has several important limitations. The data we obtained were limited to only publicly available sources. We decided against a member survey as response rates for surveys tend to be low and it is difficult to obtain information about past events. Not all programs listed a PD or DMSE in the AUPO directory which would have resulted in an undercount in each category. Likewise, Canadian programs are likely underrepresented as only a few are listed in the directory; and those that are listed may not have listed their DMSEs and PDs. Other limitations of our study include the inability to determine the academic rank of the DMSE and PD and the inability to determine academic success outside of the departments of ophthalmology, for example, holding leadership positions at the medical schools.

\section{Conclusion}

Medical student education is an important academic career path in many medical specialties. In academic ophthalmology, the career opportunities available to those interested in this academic pathway have grown substantially since 2010 , highlighting the importance that many ophthalmology departments have placed on the mission of educating medical students, the recognition of the time and energy it takes to teach, and the important role those who spend time teaching students play in their departments. As the number of DMSEs have increased, the number of women becoming DMSEs have grown. This trend does mirror the overall increase in women entering residencies. We are hopeful that the information presented in this paper will continue to shed light on leadership roles in departments of ophthalmology. In particular, we are hopeful that there will be greater numbers of women becoming PDs to increase gender equity and an increase in the number of DMSEs becoming Chairs of departments. 


\section{Financial Disclosure}

None.

\section{Conflict of Interest}

No conflicting relationship exists for any author.

\section{Acknowledgments}

We acknowledge Steven Feldon Steven Feldon, MD and Rebecca Salowe Rebecca Salowe, MSE for their assistance with the manuscript.

\section{References}

1 Association of University Professors of Ophthalmology. Association of University Professors of Ophthalmology serves, strengthen, and represents academic departments of ophthalmology. Accessed September 3, 2021 at:https://aupo.org

2 Lippa LM. Re: Albert et al.: A proposal to improve ophthalmic education in medical schools (ophthalmology 2014;121:1157-9). Ophthalmology 2014;121(12):e70

3 Mottow-Lippa L. Ophthalmology in the medical school curriculum: reestablishing our value and effecting change. Ophthalmology 2009;116(07):1235-1236, 1236.e1

4 Shah M, Knoch D, Waxman E. The state of ophthalmology medical student education in the United States and Canada, 2012 through 2013. Ophthalmology 2014;121(06):1160-1163

5 Moxon NR, Goyal A, Giaconi JA, et al. The state of ophthalmology medical student education in the United States: an update. Ophthalmology 2020;127(11):1451-1453

6 Dotan G, Qureshi HM, Gaton DD. Chairs of United States academic ophthalmology departments: a descriptive analysis and trends. Am J Ophthalmol 2018;196:26-33
7 Nettleman M, Schuster BL. Internal medicine department chairs: where they come from, why they leave, where they go. Am J Med 2007;120(02):186-190

8 US census bureau. https://www2.census.gov/geo/pdfs/mapsdata/maps/reference/us_regdiv.pdf

9 Social science statistics. Accessed September 3, 2021 at: https:// www.socscistatistics.com/

10 Salsberg E, Rockey PH, Rivers KL, Brotherton SE, Jackson GR. US residency training before and after the 1997 Balanced Budget Act. JAMA 2008;300(10):1174-1180

11 ACGME. Specialty-specific program requirements: program director qualifications. Accessed september 3, 2021 at:https:// www.acgme.org/Portals/0/PDFs/Specialty-specific\%20Requirement\%20Topics/DIO-PD_Qualifications.pdf

12 Long TR, Brown MJ, Elliott BA, Rose SH. Characteristics of anesthesiology residency program directors. J Clin Anesth 2010;22 (08):583-586

13 Mimouni M, Zayit-Soudry S, Segal O, et al. Trends in authorship of articles in major ophthalmology journals by gender, 2002-2014. Ophthalmology 2016;123(08):1824-1828

14 Xierali IM, Nivet MA, Wilson MR. Current and future status of diversity in ophthalmologist workforce. JAMA Ophthalmol 2016; 134(09):1016-1023

15 Carpenter AM, Tan SA, Costopoulos K, Cooper LA, Sarosi GA, Shaw CM. Gender diversity in general surgery residency leadership. J Surg Educ 2018;75(06):e68-e71

16 Han J, Stillings S, Hamann H, Terry R, Moy L. Gender and subspecialty of urology faculty in department-based leadership roles. Urology 2017;110:36-39

17 Fishman JE, Pang JHY, Losee JE, Rubin JP, Nguyen VT. Pathways to academic leadership in plastic surgery: a nationwide survey of program directors, division chiefs, and department chairs of plastic surgery. Plast Reconstr Surg 2018;141(06): 950e-958e 